\title{
Coexistence of Specialist and Generalist Species Is Shaped by Dispersal and Environmental Factors
}

\author{
Lucie Büchi* and Séverine Vuilleumier \\ Department of Ecology and Evolution, Biophore Building-Quartier Sorge, University of Lausanne, CH-1015, Lausanne, Switzerland \\ Submitted May 2, 2013; Accepted November 27, 2013; Electronically published March 28, 2014 \\ Online enhancement: supplementary PDFs.
}

ABSTRACt: Disentangling the mechanisms mediating the coexistence of habitat specialists and generalists has been a long-standing subject of investigation. However, the roles of species traits and environmental and spatial factors have not been assessed in a unifying theoretical framework. Theory suggests that specialist species are more competitive in natural communities. However, empirical work has shown that specialist species are declining worldwide due to habitat loss and fragmentation. We addressed the question of the coexistence of specialist and generalist species with a spatially explicit metacommunity model in continuous and heterogeneous environments. We characterized how species' dispersal abilities, the number of interacting species, environmental spatial autocorrelation, and disturbance impact community composition. Our results demonstrated that species' dispersal ability and the number of interacting species had a drastic influence on the composition of metacommunities. More specialized species coexisted when species had large dispersal abilities and when the number of interacting species was high. Disturbance selected against highly specialized species, whereas environmental spatial autocorrelation had a marginal impact. Interestingly, species richness and niche breadth were mainly positively correlated at the community scale but were negatively correlated at the metacommunity scale. Numerous diversely specialized species can thus coexist, but both species' intrinsic traits and environmental factors interact to shape the specialization signatures of communities at both the local and global scales.

Keywords: specialization, spatial autocorrelation, disturbance, interspecific competition, metacommunity, migration.

\section{Introduction}

In nature, some species exhibit narrow environmental tolerances, constraining them to particular habitats for which they are well adapted ("specialist" species), while others have broader environmental tolerances, thriving in a large diversity of habitats ("generalist" species). It is generally assumed that specialist species tend to perform better than

\footnotetext{
* Corresponding author. Present address: Agroscope, Institute for Plant Production Sciences, 1260 Nyon, Switzerland; e-mail: lucie.buchi@gmail.com.

Am. Nat. 2014. Vol. 183, pp. 612-624. (c) 2014 by The University of Chicago. 0003-0147/2014/18305-54646\$15.00. All rights reserved. DOI: $10.1086 / 675756$
}

generalist species in their optimal habitats, at the expense of their performance in other habitats (Levins 1968; Lawlor and Maynard Smith 1976; Futuyma and Moreno 1988; Wilson and Yoshimura 1994; Marvier et al. 2004; Jasmin and Kassen 2007). As a consequence, specialist species should eventually exclude generalist species in their optimal habitats (MacArthur and Levins 1964; Morris 1996).

However, the coexistence of habitat specialist and generalist species is widely observed. In addition, specialist species are currently declining worldwide at a higher rate than generalist species due to habitat loss and fragmentation (Warren et al. 2001; Julliard et al. 2004; Munday 2004; Olden et al. 2004; Rooney et al. 2004). This trend has drastic consequences with respect to species diversity, as the replacement of specialist species by generalists leads to functional homogenization (Olden et al. 2004; Olden and Rooney 2006; Clavel et al. 2010). Various mechanisms have been hypothesized to explain the maintenance and coexistence of generalist and specialist species (Débarre and Gandon 2010; Poisot 2011). It has been shown that habitat heterogeneity (in space and time) tends to favor generalist species, while specialists benefit from homogeneous habitat conditions (Smith 1982; Futuyma and Moreno 1988; Brown 1996; Kassen 2002; Marvier et al. 2004; Devictor et al. 2008; Verberk et al. 2010). Indeed, specialization on a particular habitat or resource is advantageous only when access to this habitat or resource is predictable. Generalist species settlement could also be favored when specialist species are maintained below their habitat carrying capacity, such as by environmental disturbance (Brown 1996; Morris 1996). In addition, dispersal ability and specialization have been shown to be intricately linked, and their interaction is known to influence the coexistence of specialists and generalists (Kisdi 2002; Nurmi and Parvinen 2011). In particular, dispersal limitation of specialist species could give an advantage to generalist species during colonization of new habitats. Finally, the number of competing species, and hence, the strength of interspecific competition, is also expected to play an important role in the specialization level 
of a community. Generalists are often described as opportunistic species and thus efficient in the absence of strong competitors, while specialists are more associated with $K$ strategy characteristics such as high competitive ability (Southwood 1988). It is thus expected that generalist species are favored in low-competition environments, such as in early succession stages, when the number of interacting species is low.

Both species' life-history traits (niche breadth, dispersal ability) and environmental factors (disturbance, spatial heterogeneity, and autocorrelation) are thus expected to influence the coexistence of specialist and generalist species and to favor distinct and differentiated levels of specialization in natural communities. However, while the evolution of ecological specialization has been a long-standing subject of investigation (review in Futuyma and Moreno 1988; Ravigné et al. 2009), the dynamics of the coexistence of species with different levels of specialization and dispersal abilities in heterogeneous environments has been poorly described. Indeed, often, theoretical studies on generalist-specialist species coexistence involved only a few species, a restricted number of habitat types, or discrete arbitrary levels of specialization.

We addressed these issues in a more realistic metacommunity framework, which allows for the combination of spatially explicit as well as local environmental aspects. We investigated the influences of disturbance, spatial heterogeneity, and autocorrelation, as well as of interspecific competition and species' dispersal ability on the coexistence of habitat generalist and specialist species. We also studied the effect of spatial scale (global vs. local levels) on the relationship between species richness and specialization.

\section{Methods}

\section{Model Description}

To characterize community composition and investigate the mechanisms of coexistence of habitat generalist and specialist species in heterogeneous environments, we used a spatially explicit metacommunity model (described in Büchi et al. 2009; Büchi and Vuilleumier 2012; code in Delphi Environment available by request). This model considers numerous species competing for space, which are characterized by their niche optimum $\mu_{\mathrm{s}}$, niche breadth $\sigma_{\mathrm{s}}$ and dispersal ability $\delta_{\mathrm{s}}$. Competition for settlement occurs in a grid environment composed of $625(25 \times 25)$ habitat cells. Each cell supports a local community of carrying capacity $K$ (set here to 100). The species growth rate in each community is determined by an environmental value $E_{i}$, which can vary from one cell to another. The spatial distribution of $E_{i}$ can display various degrees of spatial autocorrelation $\alpha$, derived from the sequential Gaussian simulation algorithm (Goovaerts 1999). Term $\alpha$ represents the distance above which the correlation between the environmental values of two cells falls below 0.05. An illustration of the generated spatial autocorrelation patterns given different $\alpha$ values can be found in figure 1 in Büchi and Vuilleumier (2012).

Metacommunity dynamics occur in four discrete time steps. 1, species reproduce; 2 , following reproduction, individuals can die from two distinct mortality sources: the species' annual mortality rate and the disturbance rate; 3 , juveniles could disperse; and 4 , the juveniles then compete for space to settle. All steps occur simultaneously in each cell at each time step. Reproduction depends on the local fecundity $R_{s}$, which differs among species and depends on the deviation of the local environmental value $E_{i}$ from the individual niche optimum $\mu_{\mathrm{s}}$, and on the niche breadth $\sigma_{\mathrm{s}}$ (an inverse measure of specialization) of the species (fig. 1):

$$
R_{\mathrm{s}}\left(E_{i}\right)=h \times \frac{1}{\sigma_{\mathrm{s}} \sqrt{2 \pi}} \times \exp \left[-\frac{1}{2} \times\left(\frac{E_{i}-\mu_{\mathrm{s}}}{\sigma_{\mathrm{s}}}\right)^{2}\right] .
$$

Equation (1) allows for an identical cumulative growth rate for all species, whatever their niche breadth. Term $h$, set to 10 , is a scaling factor allowing for an effective fecundity value.

Individual mortality can occur through two processes. First, species are assumed to be annual, and each adult individual dies after reproduction. Second, disturbance can cause the extinction of all species (juveniles and adults) in a local community. When disturbance occurs, at each time step, a proportion $T$ of the metacommunity (randomly chosen) is driven to extinction.

Only juvenile individuals disperse. The probability that a juvenile disperses at a distance $x$ from its birth cell is determined by a dispersal kernel $D_{\mathrm{s}}(x)$ :

$$
D_{s}(x)=\frac{1}{\delta_{s}} \times \exp \left(-\frac{x}{\delta_{s}}\right) .
$$

The shape of the kernel depends on the mean dispersal ability of the species $\delta_{\mathrm{s}}$, which can range from 0 to $\delta_{\max }$; $\delta_{\max }$ is set to $1 / 10$ of the diagonal of the grid landscape. Thus, dispersal kernel is scaled to the landscape grid size. Equation (2) determines the dispersal distance for each disperser independently, and then their dispersal direction is randomly drawn from a uniform distribution. The landscape in which dispersal occurs has periodic boundaries (torus); that is, when individuals reach the grid boundaries, they reenter the grid on the opposite edge.

Competition for space determines species settlement: juveniles of each species (resident and immigrant) have 


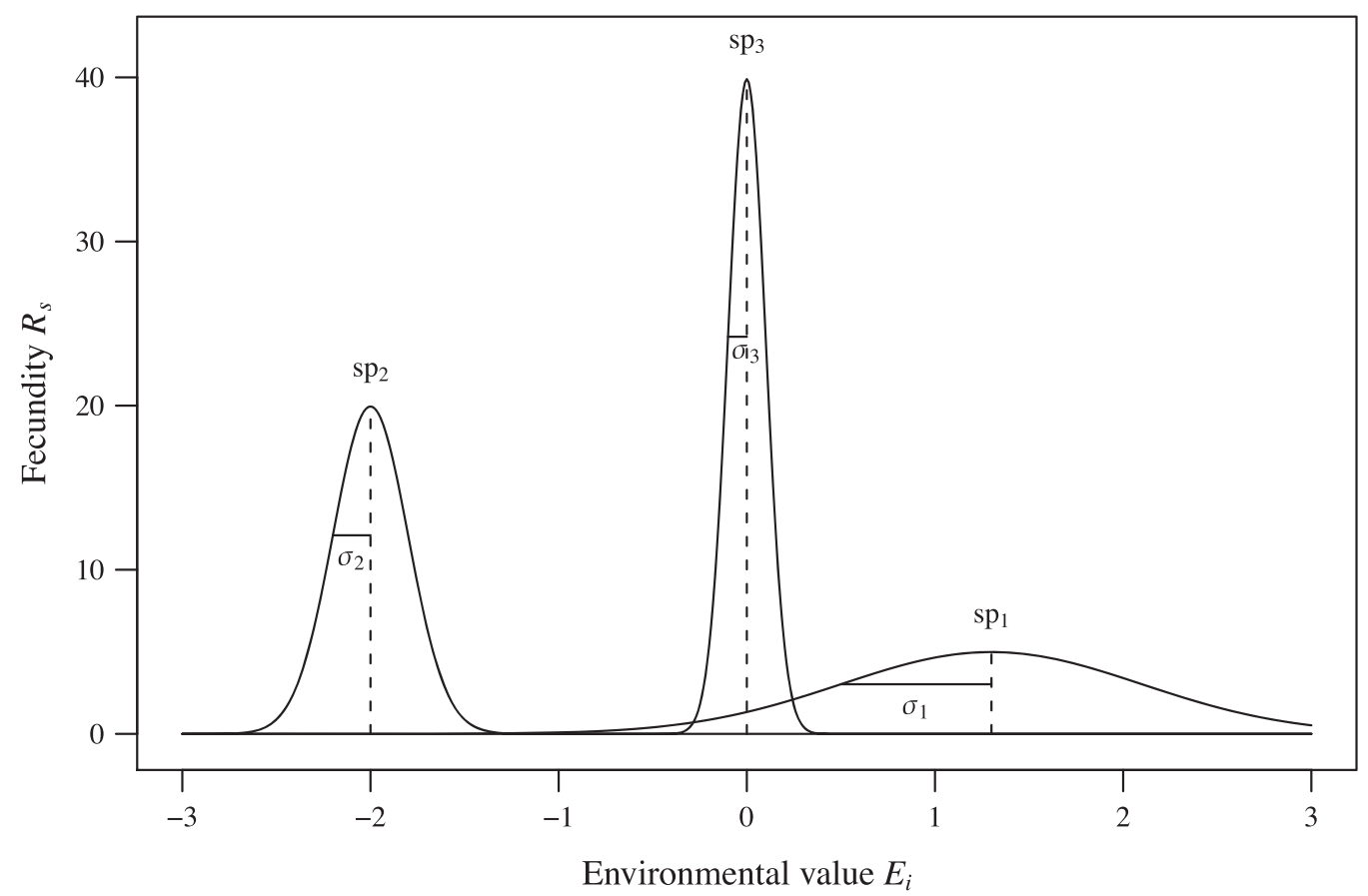

Figure 1: Fecundity function $R_{\mathrm{s}}$ (eq. [1]) in response to the environmental value $E_{i}$ for different species. In the figure are represented three species, $\mathrm{sp}_{1}, \mathrm{sp}_{2}$, and $\mathrm{sp}_{3}$, that have different niche breadths $\sigma_{\mathrm{s}}$ and niche optimums $\mu_{\mathrm{s}}$. The species with the largest niche breadth, and hence the most generalist, is $\mathrm{sp}_{1}$, while $\mathrm{sp}_{3}$ has the smallest niche breadth and is thus the most specialist. The niche breadth of $\mathrm{sp}_{2}$ is intermediate between the niche breadths of $\mathrm{sp}_{1}$ and $\mathrm{sp}_{3}$.

the same probability of settling, and a maximum of $K$ juveniles can become established at each time step. Thus, competition depends on juveniles' abundance.

\section{Simulation Setup}

We addressed the influence of four main factors on the coexistence of specialists and generalists: 1 , initial number of competing species (interspecific competition); 2, species' dispersal abilities; 3 , environmental spatial autocorrelation; and 4 , disturbance rate. Initially, the metacommunity was composed of a pool of initSp species (respectively 2, 5, 10, $25,50,100,250$, and 1,000 species per pool) in which each species had a random niche breadth $\sigma_{\mathrm{s}}$ (gradient of specialization with values between 0.01 and 1 ; fig. 1) and optimum $\mu_{\mathrm{s}}$ (values between -2.5 and 2.5; table 1). The measure of specialization is continuous and allows an investigation of a large range of community composition. Four types of species pools, differing in the dispersal strategies of the species, were considered: low dispersal (LD; $\left.\delta_{\mathrm{s}}=0.1\right)$, medium dispersal (MD; $\left.\delta_{\mathrm{s}}=0.5\right)$, high dispersal $\left(\mathrm{HD} ; \delta_{\mathrm{s}}=1.0\right)$, and random dispersal $\left(\mathrm{RD} ; \delta_{\mathrm{s}}\right.$ randomly drawn between 0 and 1 ; table 1 ). The landscape grid was heterogeneous, and three degrees of environmental spatial autocorrelation $\alpha$ were investigated: $\alpha=0$ (unstructured landscapes), $\alpha=5$ (slightly structured landscapes) and $\alpha=10$ (highly structured landscapes; table 1). Finally, three different rates of disturbance causing the extinction of local communities were considered: $T=0, T=0.01$ and $T=0.25$ (the proportion of disturbed cells, which are randomly selected; table 1 ).

Metacommunity dynamics were simulated for 1,000 time steps. This duration is sufficient to study the composition of the metacommunity in a very slow and longterm transient state (for details, see the trajectories of the metacommunity composition for all cases investigated in Supplementary Material I; Supplementary Material files IIII available online). During this time, some species became extinct and others persisted. For each simulation, a new species pool and landscape was generated, and 100 replicates were run for each scenario considered. At the beginning of each simulation, for each cell, individuals were randomly drawn from the pool of species (with replacement) until carrying capacity was reached.

At the end of each simulation, we recorded the niche optimum and breadth as well as the dispersal ability and abundance of each surviving species. Then, the number of surviving species (species richness) and the mean, minimal, and maximal niche breadth (weighted by the species' abundances) was computed at the global metacommunity 
Table 1: Parameter values used for the simulations

\begin{tabular}{llll}
\hline Parameters & Symbols & \multicolumn{1}{c}{ Phases } & \multicolumn{1}{c}{ Values } \\
\hline Niche optimum & $\mu_{\mathrm{s}}$ & Reproduction & Random [-2.5 to 2.5] \\
Niche breadth & $\sigma_{\mathrm{s}}$ & Reproduction & Random [.01-1] \\
Dispersal ability & $\delta_{\mathrm{s}}$ & Dispersal & .1 (LD), $5(\mathrm{MD}), 1.0(\mathrm{HD})$, \\
& & & random [0-1] (RD) \\
Number of competing species & initSp & & $2,5,10,25,50,100,250,1,000$ \\
Spatial autocorrelation & $\alpha$ & & $0,5,10$ \\
Disturbance rate & $T$ & Mortality & $0, .01, .25$ \\
\hline
\end{tabular}

Note: $\mathrm{LD}=$ low dispersal, $\mathrm{MD}=$ medium dispersal, $\mathrm{HD}=$ high dispersal, and $\mathrm{RD}=$ random dispersal.

level (considering all 625 cells together). We then compared the species richness and the mean, maximal, and minimal niche breadth between the different simulations to assess the influence of spatial structure, global disturbance, dispersal ability, and interspecific competition on the specialization level of the metacommunity.

We also investigated the relationship between the number of coexisting species and their mean niche breadth within each scenario studied. This relationship could be studied at the metacommunity level (comparison between different metacommunities) as well as at the local community level (comparison between different communities within a metacommunity). We thus computed Pearson coefficients of correlation between the mean niche breadth and species richness at these two spatial scales. At the metacommunity level, each correlation was performed with the 100 replicated metacommunities for each scenario $(8$ initial numbers of competing species $\times 4$ dispersal modes $\times 3$ autocorrelation ranges $\times 3$ disturbance rates $=288$ correlations). At the local community level, each correlation was performed on the 625 local communities constituting each metacommunity simulated, for a total of $288 \times 100=28,800$ correlations. Simulation outputs were analyzed using the software R, version 2.10.1 (R Development Core Team 2009).

\section{Results}

In our simulations, up to 180 species persisted together depending on the combination of parameters considered (fig. 2). Specialist and generalist species could coexist at the same time, as shown by the large standard deviation of niche breadth observed in many situations (fig. 3; Supplementary Material II).

\section{Influence of Dispersal Ability on the Coexistence of Specialists and Generalists}

The coexistence of specialists and generalists was strongly dependent on the dispersal abilities of the species that composed the metacommunity (fig. 2). Indeed, when species dispersal ability was limited, metacommunities were composed of numerous generalist species, while when species had higher dispersal abilities, they formed metacommunities composed of fewer but more specialized species (fig. 2A). Dispersal ability also impacted the maximal niche breadth values observed in the metacommunity, with many more generalist species surviving when dispersal was limited compared to the cases where species had higher dispersal abilities (fig. $2 B$ ). Interestingly, although the average niche breadth value was similar when species had either a large variability of dispersal abilities (RD) or had identical intermediate dispersal abilities (MD), those dispersal abilities had different influences on the coexistence of species. Random dispersal allowed for the persistence of species with larger niche breadths (fig. 2), and niche breadth variance was higher in the resulting metacommunity, allowing for the coexistence of species with more diverse specialization levels (fig. 3).

\section{Influence of Interspecific Competition on the Coexistence of Specialists and Generalists}

The number of species initially competing had an important impact on the composition of the metacommunities (fig. 2). As expected, the final number of coexisting species increased with the initial number of competing species. However, the relative proportion of species that were eliminated from the metacommunity strongly increased with the number of competing species. The number of competing species also strongly affected the niche breadth. As the number of competing species increased, the mean and variance of niche breadth drastically decreased, from 0.5 to approximately 0.1 for the mean (fig. 3 and table $S 1$ in Supplementary Material III). This change occurred through the gradual disappearance of the most generalist species (fig. $2 B$ ) and through a transition in the niche breadth for which the highest abundance was reached (see Supplementary Material II for species abundances in function of their niche breadth). 


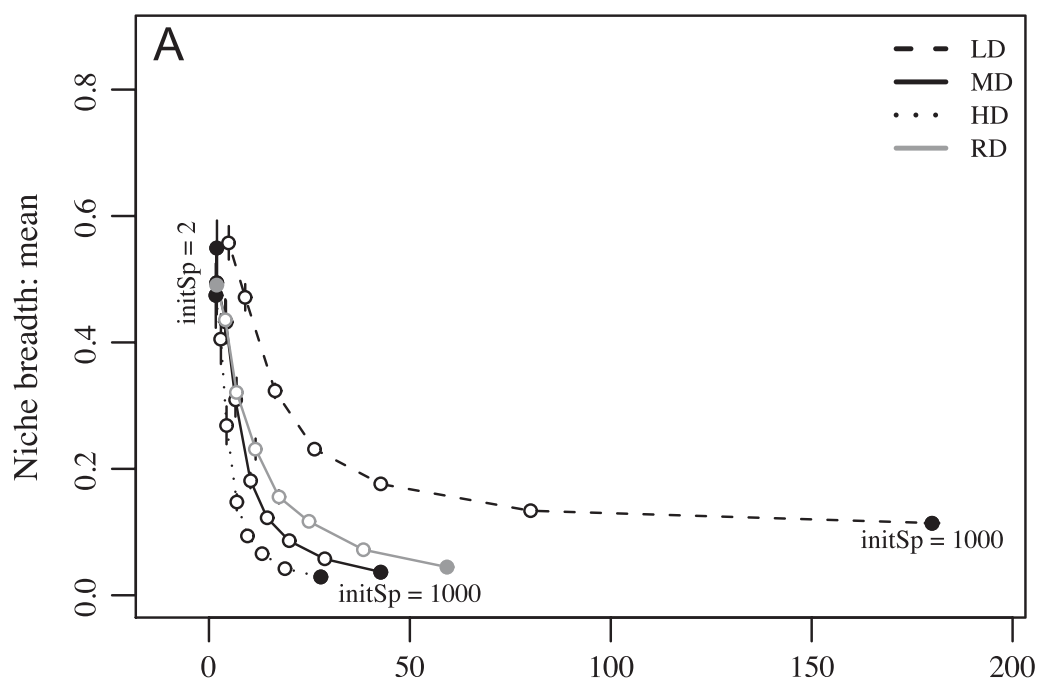

Number of coexisting species

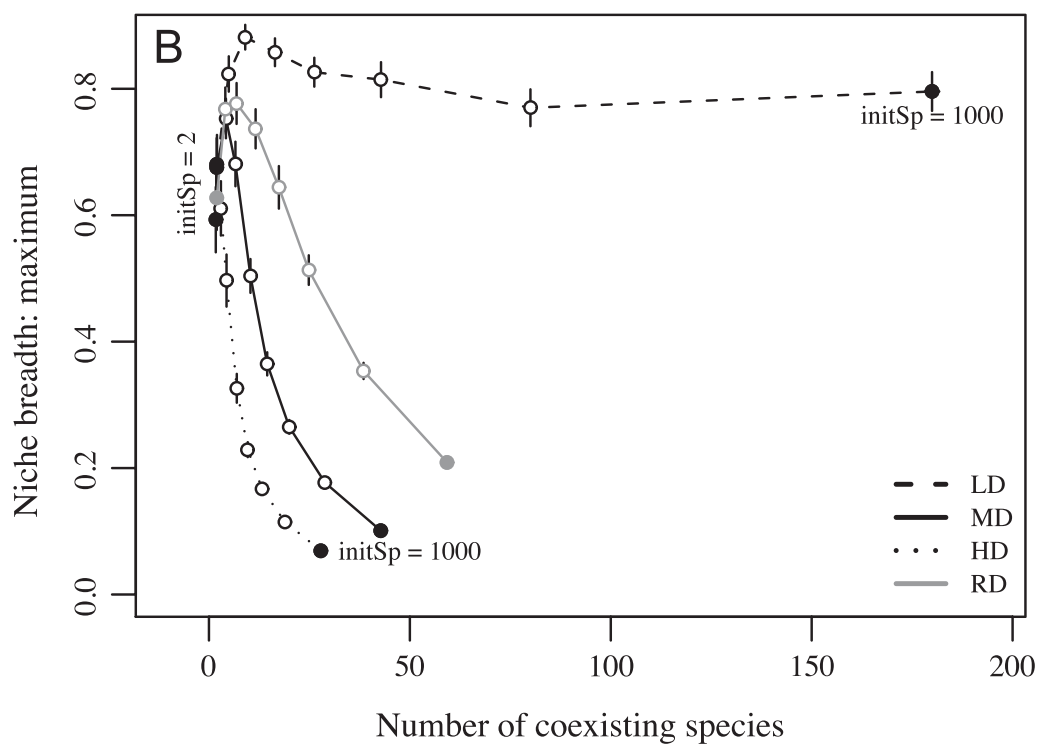

Figure 2: Metacommunity weighted mean niche breadth $(A)$ and maximal niche breadth $(B)$, as a function of the number of coexisting species, in uncorrelated and undisturbed environments $(\alpha=0$ and $T=0)$. Results are presented for different values of the initial number of competing species (see values in table 1) and of species dispersal ability, $\delta_{\mathrm{s}}=0.1$ (low dispersal [LD], dashed black line), $\delta_{\mathrm{s}}=0.5$ (medium dispersal [MD], solid black line), $\delta_{\mathrm{s}}=1.0$ (high dispersal [HD], dotted black line) and variable dispersal ability $\delta_{\mathrm{s}}=$ random value between 0 and 1 (random dispersal $[\mathrm{RD}]$, solid gray line). Each dot represents the mean of the $n=100$ replicates. The error bars corresponds to $\pm 2 \mathrm{SE}$.

\section{Influence of Disturbance and Spatial Autocorrelation on the Coexistence of Specialists and Generalists}

At the metacommunity level, the increase of the disturbance rate diminished the number of coexisting species, regardless of the species' dispersal abilities (fig. 4). In addition, increased disturbance tended to favor more gen- eralist species, particularly when species had strongly limited dispersal. The effects of spatial autocorrelation on the number and specialization level of the coexisting species were less straightforward, as these effects depended on the dispersal abilities of the species that composed the metacommunity (fig. 4). 

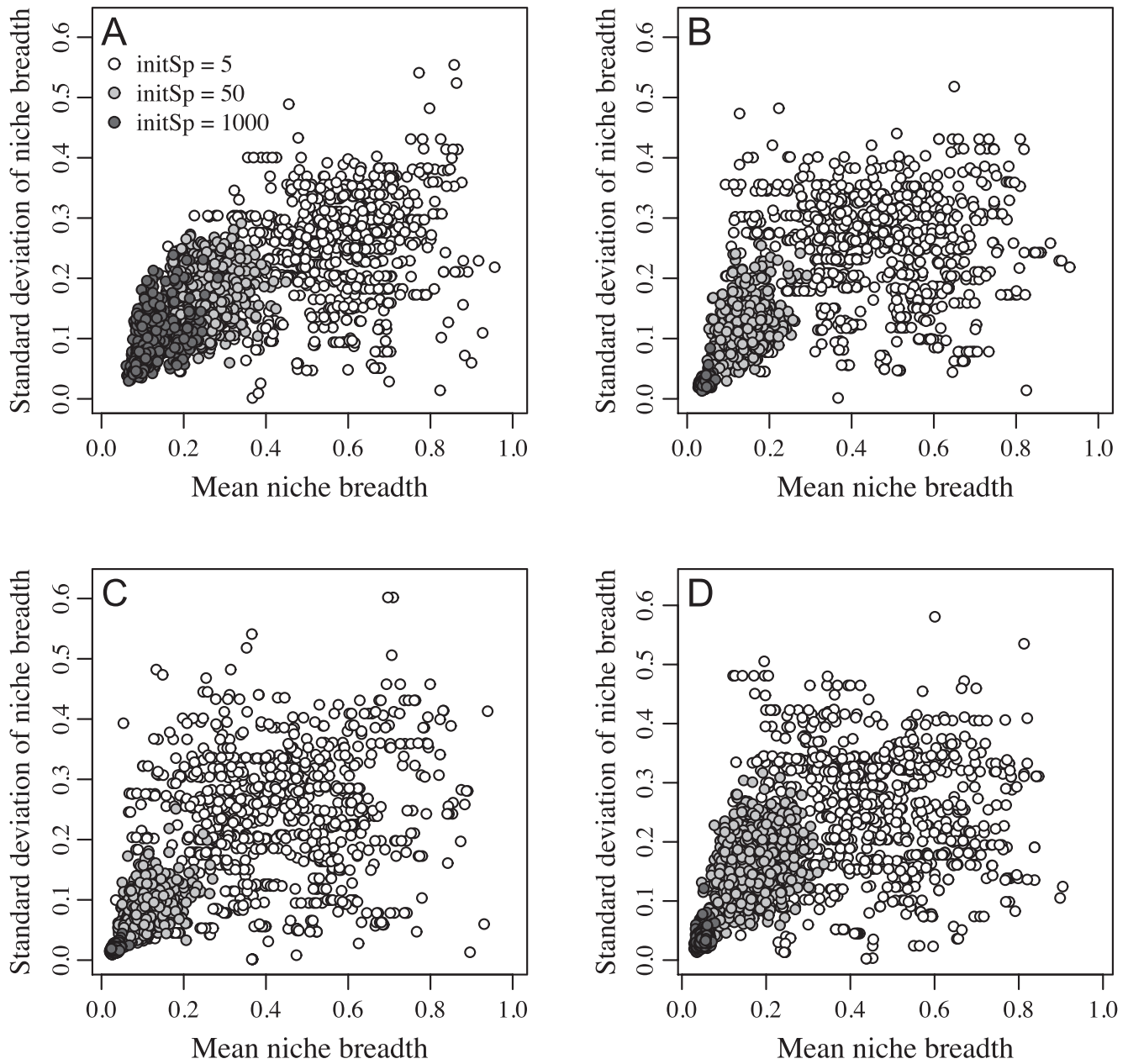

Figure 3: Standard deviation of niche breadth as a function of the weighted mean niche breadth at the metacommunity level. Each dot represents one replicate. Results are presented for three different values of the initial number of competing species (initSp $=5,50$, and 1,000). $A$, Dispersal ability $\delta_{\mathrm{s}}=0.1$ (low dispersal). B, Dispersal ability $\delta_{\mathrm{s}}=0.5$ (medium dispersal). $C$, Dispersal ability $\delta_{\mathrm{s}}=1.0$ (high dispersal). $D$, Variable dispersal ability, $\delta_{\mathrm{s}}=$ random value between 0 and 1 (random dispersal).

When species had low dispersal ability, spatial autocorrelation decreased the mean niche breadth and thus favored more specialist species (fig. 4). Additionally, environmental autocorrelation decreased the number of coexisting species, except in situations where the disturbance rate was high $(T=0.25)$. When species had higher dispersal abilities, more species coexisted with increasing environmental spatial autocorrelation, and coexisting species had slightly higher mean niche breadth, thus being more generalist species than in uncorrelated environments (fig. 4).

In addition to its effects on the mean niche breadth, disturbance rate and spatial autocorrelation also affected the range of species' niche breadth in the metacommunity. Whereas the most specialized species almost always survived in each scenario, the survival of the most generalist species strongly depended on all of these factors.

\section{Influence of Spatial Scale on the Coexistence of Specialists and Generalists}

The relationship between the number of coexisting species and their mean niche breadth differed when studied at the local community level (comparison of different communities within a metacommunity) or at the metacommunity level (comparison of different metacommunities). At the metacommunity level, species richness and mean niche breadth were negatively correlated for most of the parameter combinations considered (214 out of 288 cases; fig. 5A, 5C). Thus, as the number of species that coexisted increased, so did their level of specialization. Exceptions occurred when the number of competing species was large or in autocorrelated environments, when species had limited dispersal abilities. In contrast, at the community level, species richness 


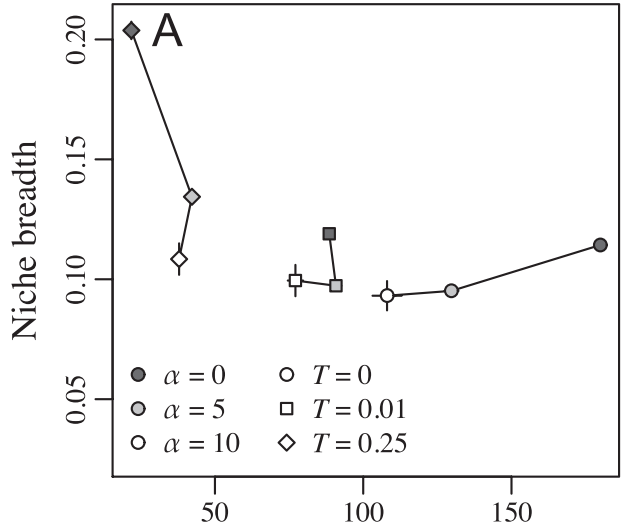

Number of coexisting species

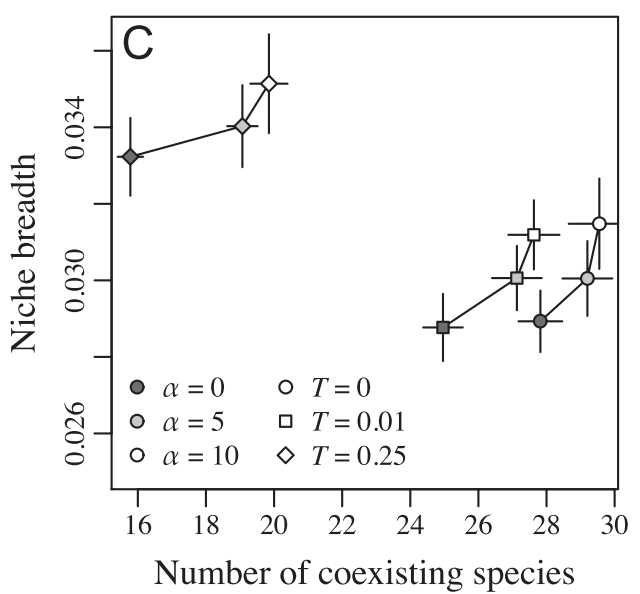

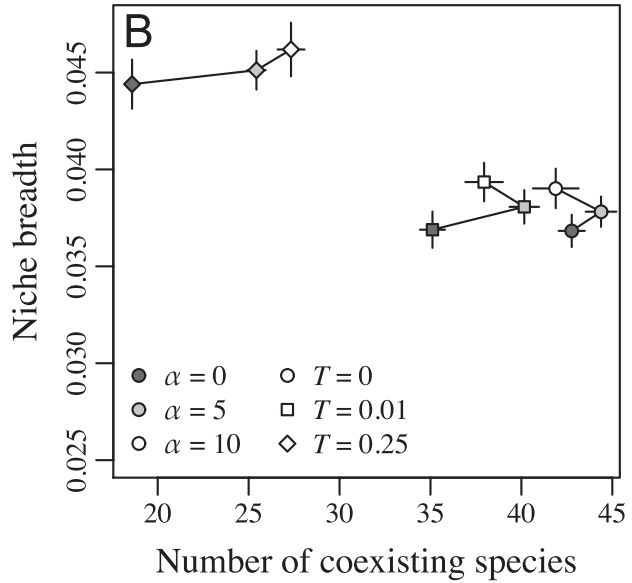

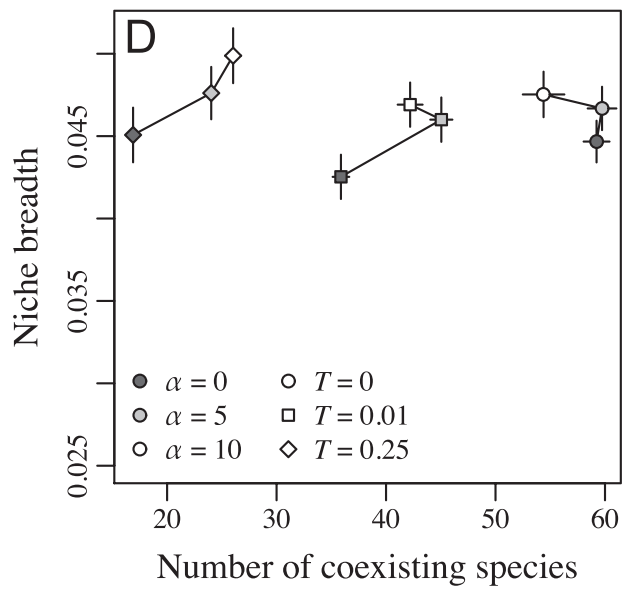

Figure 4: Weighted mean metacommunity niche breadth as a function of the number of species coexisting in the metacommunities. Results are presented for three values of spatial autocorrelation $\alpha$ and disturbance rate $T$, for an initial pool size of 1,000 species. A, Dispersal ability $\delta_{\mathrm{s}}=0.1$ (low dispersal). $B$, Dispersal ability $\delta_{\mathrm{s}}=0.5$ (medium dispersal). $C$, Dispersal ability $\delta_{\mathrm{s}}=1.0$ (high dispersal). $D$, Variable dispersal ability, $\delta_{\mathrm{s}}=$ random value between 0 and 1 (random dispersal). Each dot represents the mean of the $n=100$ replicates. The error bars corresponds to $\pm 2 \mathrm{SE}$.

was mainly positively correlated with mean niche breadth $(22,906$ out of 28,800 cases; fig. $5 B, 5 D)$, except for some cases when the initial number of competing species was small. Thus, as the number of species that coexisted increased, their level of specialization decreased.

\section{Discussion}

Our results have demonstrated that the coexistence of specialists and generalists in a metacommunity depended strongly on the species' dispersal abilities, as well as on the initial species richness, that is, the initial number of competing species. Generalist species were favored when dispersal was limited and when interspecific competition was low, in disturbed nonautocorrelated environments. In contrast, specialist species benefited from stable conditions, with low disturbance intensity.

\section{Interaction of Dispersal and Environmental Structure}

Our results have shown that species' dispersal abilities shape the level of specialization and richness of metacommunities. When species' dispersal ability is limited, a large number of species can coexist in the metacommunity. Indeed, dispersal limitation prevents the displacement of locally poor competitors by locally higher competitors and promotes the coexistence of species (Hurtt and Pacala 1995; Levine and Rees 2002). This observation is especially true for specialist species, as their narrow environmental tolerance creates a barrier that prevents them from reach- 

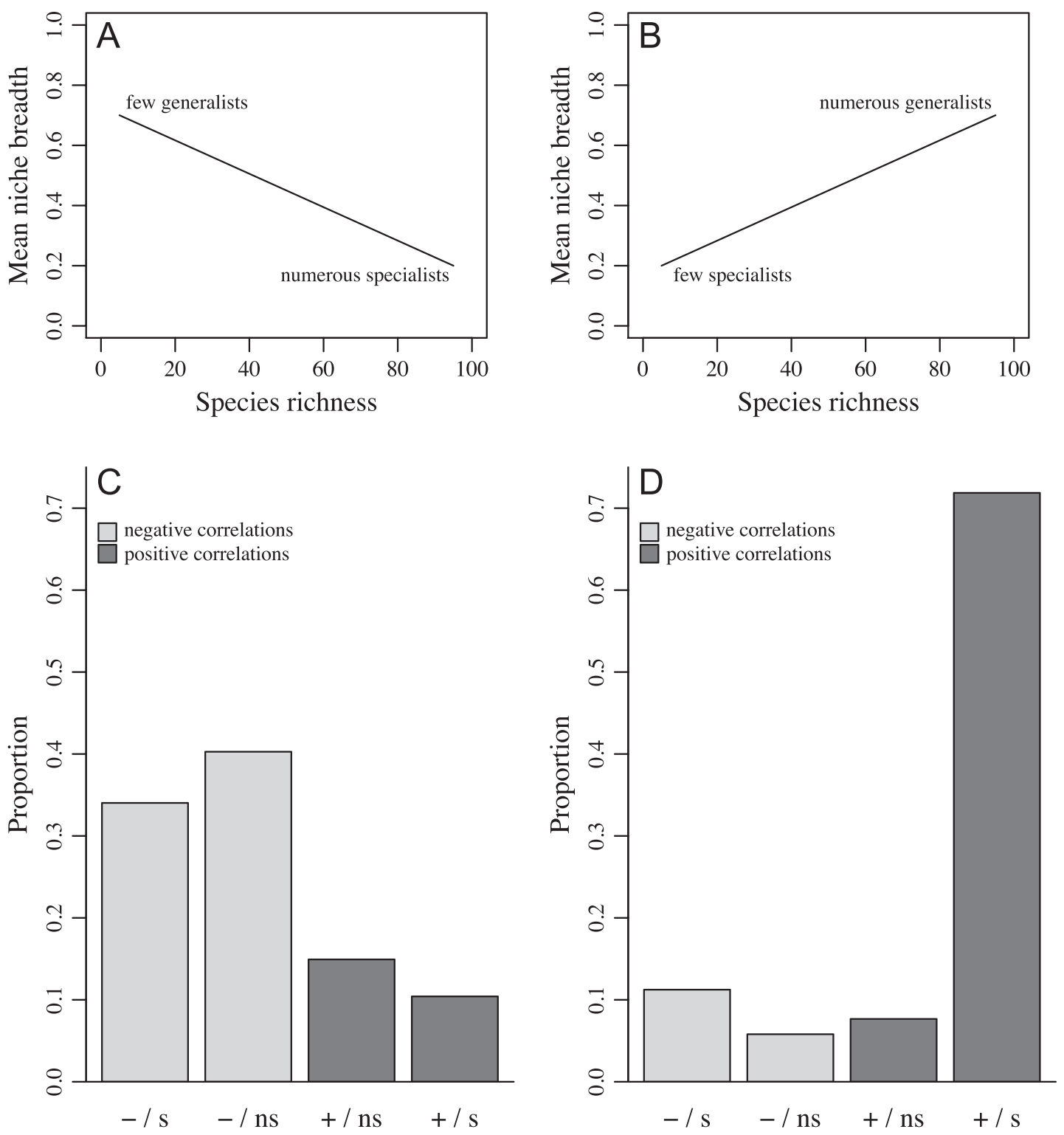

Figure 5: $A, B$, Illustration of the observed relationships between species' richness and mean niche breadth: $A$, negative correlation; $B$, positive correlation. $C, D$, Proportion of positive and negative correlations between species richness (number of coexisting species) and mean niche breadth (inverse of specialization) at the metacommunity level $(C)$ and at the local community level $(D)$. The bars represent the proportion of significant negative correlations $(-/ \mathrm{s})$, nonsignificant negative correlations $(-/ \mathrm{n})$, nonsignificant positive correlations $(+/$ ns), and significant positive correlations $(+/ \mathrm{s})$. Significance is assessed at $P<.05$.

ing other favorable habitats. In contrast, generalists benefit from their higher environmental tolerance, which allows them to disperse gradually to neighboring habitats. We also show that environmental heterogeneity reinforces this process, while, in spatially autocorrelated environments, the clustering of habitats alleviates the dispersal limitation of specialists. This trend confirms that generalist species are favored by environmental heterogeneity (Kassen 2002; Swihart et al. 2003; Henle et al. 2004; Marvier et al. 2004;
Swihart et al. 2006; Devictor et al. 2008). However, this finding is observed only when specialist species have restricted dispersal abilities. When specialist species have high dispersal abilities, they can reach their distant optimal habitats and can thus outcompete the less adapted generalist species. Our results are thus in opposition to several studies that argue that high dispersal rates are detrimental to specialists (Brown and Pavlovic 1992; Ronce and Kirkpatrick 2001; Kisdi 2002; Parvinen and Egas 2004). These 
opposing results can be discussed in the light of the "specialists' paradox" (Poisot et al. 2011; Stevens et al. 2012). While specialist species need large dispersal abilities to find favorable habitats, which are usually sparse, they benefit from being philopatric once settled in a suitable habitat, as their chance of landing in another suitable habitat is low (Kisdi 2002; Parvinen and Egas 2004; Jocque et al. 2010). In natural habitats, the likely evolution of specialization in homogeneous and stable habitats (Futuyma and Moreno 1988; Brown 1996; Kassen 2002; Jasmin and Kassen 2007) has thus selected for decreased dispersal abilities, preventing specialists from efficiently coping with habitat loss and fragmentation. This reasoning confirms that maintaining connectivity between habitat patches is crucial for the conservation of specialist species (Brückmann et al. 2010).

\section{Initial Species Richness, Interspecific Competition, and Colonization of New Habitats}

The initial species richness (or the initial number of competing species), although seldom considered in previous studies, appears to have a drastic impact on the metacommunity specialization. The presence of numerous highly specialized species increases with the initial number of species. This outcome increases the number of juveniles in the metacommunity and the competition among species. The initial species richness also promotes a facilitation effect between specialists, which directly impacts the ratio of specialists to generalists. Indeed, each specialist contributes to the lowering of the generalists' global abundance through their local fitness advantage, thus preventing generalist settlement and diminishing their migration. When the initial species richness is low, most of the species are maintained in the metacommunity, and generalists have an advantage over specialists, as they could occupy a larger number of habitats. When the initial species richness increases, the proportion of generalist species decreases, as each habitat is occupied by specialized species. The generalist species are thus systematically outcompeted by more specialized species. This scenario demonstrates that interspecific competition has a critical influence on the level of metacommunity specialization; when interspecific competition is strong, generalist species are gradually eliminated (Futuyma and Moreno 1988). This phenomenon is likely to play an important role in colonization processes and could help explain the current rise of generalist species. Indeed, habitat destruction and changes induced by climatic and anthropogenic factors tend to open new habitats and offer opportunities for opportunistic generalist species to profit from the relaxing of interspecific competition.

\section{Disturbance and Functional Homogenization}

We demonstrated that an increase in disturbance rate decreases the number of coexisting species while increasing the mean niche breadth of the metacommunity. Disturbance thus favors generalist species. This finding can be explained by the high extinction risk of specialized species due to disturbance, in a landscape where their habitats are scarce. This risk is reduced for generalist species, as they can colonize a larger amount of empty habitats due to their higher environmental tolerance. This finding is in agreement with many studies showing a greater sensitivity of specialist species to disturbance (Jonsen and Fahrig 1997; Marvier et al. 2004; Devictor et al. 2008; Devictor and Robert 2009) and with the observation that specialist species have increased extinction risk due to the degradation of habitats (Foufopoulos and Ives 1999; Henle et al. 2004; Colles et al. 2009).

However, other studies have questioned the generality of this outcome, showing that habitat disturbance and degradation could sometimes favor specialist species (Parvinen and Egas 2004; Attum et al. 2006; Nurmi and Parvinen 2008). This outcome could apply when the specialist-generalist trade-off affects the growth rate of species. In this case, generalists have a lower growth rate and so are more susceptible to disappearing when disturbance is frequent (Parvinen and Egas 2004; Nurmi and Parvinen 2008). Specialists can also be favored when the degradation of the habitat leads to harsher environmental conditions in which only species adapted to such extreme conditions can persist (Attum et al. 2006). This effect can be related to the observation that stressful habitats are often occupied by species that are specially adapted to such extreme conditions (Fridley et al. 2007; Boulangeat et al. 2012). In the latter case, disturbance acts as an important environmental filter (Chase 2007). In addition, here, disturbance reduces the range of niche breadth values, that is, the diversity of specialization strategies, of the species coexisting in communities. Consequently, we observed a functional homogenization of the community, as coexisting species are more similar (less variation) in terms of niche breadth when disturbance is strong. This phenomenon deserves further investigation, as functional homogenization is becoming increasingly recognized as having important ecological and evolutionary repercussions (Olden et al. 2004; Olden and Rooney 2006; Clavel et al. 2010; Davey et al. 2012).

\section{Community versus Metacommunity Scale}

Many studies have reported that species-rich assemblies contain specialized species and species-poor assemblies contain more generalist species (MacArthur 1955; Mac- 
Arthur et al. 1966; Lister 1976; Schoener 1977; Abbott 1980; Grant 1986; Kolasa and Li 2003; Carnicer et al. 2008; Weiner and Xiao 2012). Our results, at the metacommunity level, confirm this negative relationship between species richness and niche breadth. This relationship can be explained by the fact that highly specialist species occupy a limited part of the environment in which they are well adapted, allowing for the coexistence of numerous specialist species through niche partitioning. In contrast, generalist species, with their broad environmental tolerance, can colonize and settle in many environments. However, they are maintained at lower abundance compared to specialist species due to their low fecundity. Generalists' low abundance makes them more prone to exclusion than specialists, thus reducing global species richness.

However, we demonstrated that locally, at the community level, species richness is positively correlated with niche breadth. Thus, species-rich communities contain more generalist individuals, whereas species-poor communities are more specialized. Similar relationships were observed by Clavero and Brotons (2010), Filippi-Codaccioni et al. (2010), and Davey et al. (2012), who found a positive relationship between species richness and niche breadth for bird communities, as well as in the models developed by Steiner and Köhler (2003) and Yamauchi and Miki (2009). This pattern can be explained by the fact that specialist species are locally more productive (higher per capita fitness) than generalists and quickly occupy and fill the habitats, preventing the settlement of other immigrating species. In opposition, the per capita fitness of generalists is lower, allowing for more species to coexist, with lower abundances.

The relationship between specialization and species richness is thus scale dependent. In our study, the community level corresponds to a unique environmental condition, whereas the metacommunity level encompasses a whole gradient of environmental conditions. In addition, the transition between the community and metacommunity levels is strongly mediated by the dispersal of species. Indeed, in one homogeneous community, the coexistence of species relies on the presence of immigrants. Making explicit the spatial scale at which the relationship between species richness and level of specialization is estimated is thus of crucial importance to better understand why different studies have reported divergent trends between species richness and specialization

To have a better understanding of the relationship between species richness and niche breadth in natural community, further empirical data are needed, such as those provided by Filippi-Codaccioni et al. (2010). Such data would be of crucial importance, as the complex relationship between species richness, niche breadth, and spatial scale can also have important conservation implications (Reitalu et al. 2012).

\section{Model Assumptions}

The species life cycle and traits studied are representative of annual plants. Having a metacommunity composed of species with generation overlap is expected to alter interand intraspecific competition and the dynamics of species richness; residents might have an advantage over migrants, and adults of some species might be maintained even when their juveniles are outcompeted by other juveniles. In this study, we investigated the coexistence of species when species have all possible values of dispersal ability, niche breadth, and niche optimum. This approach allows for the identification of all combinations of traits that contribute to a species' survival in the metacommunity. However, trade-offs between traits are common in nature and can drive the coexistence of species (see review in Kneitel and Chase 2004). Further investigations would benefit from the integration of correlations between species traits, evolution, and coevolution. We also assumed that the integral of the reproduction function is equal for all species; that is, species have on average the same fecundity over the landscape. This assumption allows for a better comparison of species fitness. However, it may not be always true in nature, as trade-offs with other life-history traits could result in unequal fecundity.

The relationship between fecundity and niche breadth, given a niche optimum, is fully described by the fecundity function (eq. [1]; fig. 1). It can be shown that this function defines a nonlinear relationship between the fitness of species in two different habitats. Nonlinearity in such fitness functions is known to be important for coexistence (Débarre and Gandon 2010); thus, future investigations would benefit from the study of a larger range of fitness functions. Finally, our spatially explicit simulation model investigates through in silico experiments how the composition of a community can be shaped by species' traits and environmental factors. To disentangle the relative importance of each process identified here, analytical investigations using mutual invasion theory (as in Débarre and Gandon 2010; Samia and Lutscher 2010) could be advantageously used.

\section{Conclusion}

Two opposing perspectives emerge when dealing with generalist-specialist coexistence. First, specialists are predicted to be more productive than generalists in their optimal habitats and are thus likely to outcompete generalists in most habitats. This trend would eventually lead to a clear dominance of specialists (Berenbaum 1996; Egas et al. 2005) and thus question the maintenance of generalist 
species (Abrams 2006; Ravigne et al. 2009). On the other hand, many studies have reported a global decrease in specialist species, pointing to their increased sensitivity to degraded and fragmented habitats and thus raising the question of specialists' survival in our changing world (Tilman 1994; Travis 2003; Colles et al. 2009; Atkins and Travis 2010).

The future coexistence of specialist and generalist species in the face of the current increasing changes in species' environments and habitats is thus a challenging issue. Our results showed that numerous species with a broad range of specialization values can coexist at the metacommunity level. The most favorable situation for generalist species is when all species suffer from dispersal limitation in a highly disturbed, nonautocorrelated environment. In this case, dispersal limitation and the heterogeneous environment prevent specialists from spreading into all of their optimal habitats, and the high disturbance rate strongly affects species occupying highly localized habitats. In contrast, specialists benefit from high dispersal ability and undisturbed environments. Thus, the future survival of specialist species would most likely be promoted through the maintenance of high connectivity between habitats, allowing them to sustain viable populations and limiting risks of extinction through environmental stochasticity.

\section{Acknowledgments}

We thank Editor-in-Chief J. L. Bronstein, Associate Editor M. A. Leibold, and three anonymous reviewers for their very valuable comments; as well as N. Perrin and S. Rasmann and for their comments on previous versions of the manuscript. This work was funded by the Swiss National Science Foundation, grant 31003A-112511/2 and, for S.V., PZ00P3-139421/1.

\section{Literature Cited}

Abbott, I. 1980. Theories dealing with the ecology of landbirds on islands. Advances in Ecological Research 11:329-371.

Abrams, P. A. 2006. The prerequisites for and likelihood of generalistspecialist coexistence. American Naturalist 167:329-342.

Atkins, K. E., and J. M. J. Travis. 2010. Local adaptation and the evolution of species' ranges under climate change. Journal of Theoretical Biology 266:449-457.

Attum, O., P. Eason, G. Cobbs, and S. M. B. El Din. 2006. Response of a desert lizard community to habitat degradation: do ideas about habitat specialists/generalists hold? Biological Conservation 133: 52-62.

Berenbaum, M. R. 1996. Introduction to the symposium: on the evolution of specialization. American Naturalist 148(suppl.):S78S83.

Boulangeat, I., S. Lavergne, J. Van Es, L. Garraud, and W. Thuiller.
2012. Niche breadth, rarity and ecological characteristics within a regional flora spanning large environmental gradients. Journal of Biogeography 39:204-214.

Brown, J. S. 1996. Coevolution and community organization in three habitats. Oikos 75:193-206.

Brown, J. S., and N. B. Pavlovic. 1992. Evolution in heterogeneous environments: effects of migration on habitat specialization. Evolutionary Ecology 6:360-382.

Brückmann, S. V., J. Krauss, and I. Steffan-Dewenter. 2010. Butterfly and plant specialists suffer from reduced connectivity in fragmented landscapes. Journal of Applied Ecology 47:799-809.

Büchi, L., P. A. Christin, and A. H. Hirzel. 2009. The influence of environmental spatial structure on the life-history traits and diversity of species in a metacommunity. Ecological Modelling 220: 2857-2864.

Büchi, L., and S. Vuilleumier. 2012. Dispersal strategies, few dominating or many coexisting: the effect of environmental spatial structure and multiple sources of mortality. PLoS ONE 7:e34733.

Carnicer, J., L. Brotons, D. Sol, and M. de Caceres. 2008. Random sampling, abundance-extinction dynamics and niche-filtering immigration constraints explain the generation of species richness gradients. Global Ecology and Biogeography 17:352-362.

Chase, J. M. 2007. Drought mediates the importance of stochastic community assembly. Proceedings of the National Academy of Sciences of the USA 104:17430-17434.

Clavel, J., R. Julliard, and V. Devictor. 2010. Worldwide decline of specialist species: toward a global functional homogenization? Frontiers in Ecology and the Environment 9:222-228.

Clavero, M., and L. Brotons. 2010. Functional homogenization of bird communities along habitat gradients: accounting for niche multidimensionality. Global Ecology and Biogeography 19:684696.

Colles, A., L. H. Liow, and A. Prinzing. 2009. Are specialists at risk under environmental change? neoecological, paleoecological and phylogenetic approaches. Ecology Letters 12:849-863.

Davey, C. M., D. E. Chamberlain, S. E. Newson, D. G. Noble, and A. Johnston. 2012. Rise of the generalists: evidence for climate driven homogenization in avian communities. Global Ecology and Biogeography 21:568-578.

Débarre, F., and S. Gandon. 2010. Evolution of specialization in a spatially continuous environment. Journal of Evolutionary Biology 23:1090-1099.

Devictor, V., R. Julliard, and F. Jiguet. 2008. Distribution of specialist and generalist species along spatial gradients of habitat disturbance and fragmentation. Oikos 117:507-514.

Devictor, V., and A. Robert. 2009. Measuring community responses to large-scale disturbance in conservation biogeography. Diversity and Distributions 15:122-130.

Egas, M., M. W. Sabelis, and U. Dieckmann. 2005. Evolution of specialization and ecological character displacement of herbivores along a gradient of plant quality. Evolution 59:507-520.

Filippi-Codaccioni, O., V. Devictor, Y. Bas, and R. Julliard. 2010. Toward more concern for specialisation and less for species diversity in conserving farmland biodiversity. Biological Conservation 143:1493-1500.

Foufopoulos, J., and A. R. Ives. 1999. Reptile extinctions on landbridge islands: life-history attributes and vulnerability to extinction. American Naturalist 153:1-25.

Fridley, J. D., D. B. Vandermast, D. M. Kuppinger, M. Manthey, and R. K. Peet. 2007. Co-occurrence based assessment of habitat gen- 
eralists and specialists: a new approach for the measurement of niche width. Journal of Ecology 95:707-722.

Futuyma, D. J., and G. Moreno. 1988. The evolution of ecological specialization. Annual Review of Ecology and Systematics 19:207233.

Goovaerts, P. 1999. Impact of the simulation algorithm, magnitude of ergodic fluctuations and number of realizations on the spaces of uncertainty of flow properties. Stochastic Environmental Research and Risk Assessment 13:161-182.

Grant, P. R. 1986. Ecology and evolution of Darwin's finches. Princeton University Press, Princeton, NJ.

Henle, K., K. F. Davies, M. Kleyer, C. Margules, and J. Settele. 2004. Predictors of species sensitivity to fragmentation. Biodiversity and Conservation 13:207-251.

Hurtt, G. C., and S. W. Pacala. 1995. The consequences of recruitment limitation: reconciling chance, history and competitive differences between plants. Journal of Theoretical Biology 176:1-12.

Jasmin, J. N., and R. Kassen. 2007. On the experimental evolution of specialization and diversity in heterogeneous environments. Ecology Letters 10:272-281.

Jocque, M., R. Field, L. Brendonck, and L. De Meester. 2010. Climatic control of dispersal-ecological specialization trade-offs: a metacommunity process at the heart of the latitudinal diversity gradient? Global Ecology and Biogeography 19:244-252.

Jonsen, I. D., and L. Fahrig. 1997. Response of generalist and specialist insect herbivores to landscape spatial structure. Landscape Ecology 12:185-197.

Julliard, R., F. Jiguet, and D. Couvet. 2004. Common birds facing global changes: what makes a species at risk? Global Change Biology 10:148-154.

Kassen, R. 2002. The experimental evolution of specialists, generalists, and the maintenance of diversity. Journal of Evolutionary Biology $15: 173-190$

Kisdi, E. 2002. Dispersal: risk spreading versus local adaptation. American Naturalist 159:579-596.

Kneitel, J. M., and Chase J. M. 2004. Trade-offs in community ecology: linking spatial scales and species coexistence. Ecology Letters 7:69-80.

Kolasa, J., and B. L. Li. 2003. Removing the confounding effect of habitat specialization reveals the stabilizing contribution of diversity to species variability. Proceedings of the Royal Society B: Biological Sciences 270:S198-S201.

Lawlor, L. R., and J. Maynard Smith. 1976. Co-evolution and stability of competing species. American Naturalist 110:79-99.

Levine, J. M., and M. Rees. 2002. Coexistence and relative abundance in annual plant assemblages: the roles of competition and colonization. American Naturalist 160:452-467.

Levins, R. 1968. Evolution in changing environments. Princeton University Press, Princeton, NJ.

Lister, B. C. 1976. Nature of niche expansion in West Indian Anolis lizard. 2. Evolutionary components. Evolution 30:677-692.

MacArthur, R. H. 1955. Fluctuations of animal populations, and a measure of community stability. Ecology 36:533-536.

MacArthur, R. H., and R. Levins. 1964. Competition, habitat selection, and character displacement in patchy environment. Proceedings of the National Academy of Sciences of the USA 51:12071210.

MacArthur, R. H., H. Recher, and M. Cody. 1966. On the relation between habitat selection and species diversity. American Naturalist 100:319-332.
Marvier, M., P. Kareiva, and M. G. Neubert. 2004. Habitat destruction, fragmentation, and disturbance promote invasion by habitat generalists in a multispecies metapopulation. Risk Analysis 24:869878 .

Morris, D. W. 1996. Coexistence of specialist and generalist rodents via habitat selection. Ecology 77:2352-2364.

Munday, P. L. 2004. Habitat loss, resource specialization, and extinction on coral reefs. Global Change Biology 10:1642-1647.

Nurmi, T., and K. Parvinen. 2008. On the evolution of specialization with a mechanistic underpinning in structured metapopulations. Theoretical Population Biology 73:222-243.

2011. Joint evolution of specialization and dispersal in structured metapopulations. Journal of Theoretical Biology 275:78-92.

Olden, J. D., N. L. Poff, M. R. Douglas, M. E. Douglas, and K. D. Fausch. 2004. Ecological and evolutionary consequences of biotic homogenization. Trends in Ecology and Evolution 19:18-24.

Olden, J. D., and T. P. Rooney. 2006. On defining and quantifying biotic homogenization. Global Ecology and Biogeography 15:113120.

Parvinen, K., and M. Egas. 2004. Dispersal and the evolution of specialisation in a two-habitat type metapopulation. Theoretical Population Biology 66:233-248.

Poisot, T., J. D. Bever, A. Nemri, P. H. Thrall, and M. E. Hochberg. 2011. A conceptual framework for the evolution of ecological specialisation. Ecology Letters 14:841-851.

Ravigné, V., U. Dieckmann, and I. Olivieri. 2009. Live where you thrive: joint evolution of habitat choice and local adaptation facilitates specialization and promotes diversity. American Naturalist 174:E141-E169.

R Development Core Team. 2009. R: a language and environment for statistical computing. R Foundation for Statistical Computing, Vienna.

Reitalu, T., O. Purschke, L. J. Johansson, K. Hall, M. T. Sykes, and H. C. Prentice. 2012. Responses of grassland species richness to local and landscape factors depend on spatial scale and habitat specialization. Journal of Vegetation Science 23:41-51.

Ronce, O., and M. Kirkpatrick. 2001. When sources become sinks: migrational meltdown in heterogeneous habitats. Evolution 55: $1520-1531$

Rooney, T. P., S. M. Wiegmann, D. A. Rogers, and D. M. Waller. 2004. Biotic impoverishment and homogenization in unfragmented forest understory communities. Conservation Biology 18: 787-798.

Samia, Y., and F. Lutscher. 2010. Coexistence and spread of competitors in heterogeneous landscapes. Bulletin of Mathematical Biology 72: 2089-2112.

Schoener, T. W. 1977. Competition and the niche in reptiles. Pages 35-136 in C. Gans and D. W. Tinkle, eds. Biology of the Reptilia. Academic Press, New York.

Smith, E. P. 1982. Niche breadth, resource availability, and inference. Ecology 63:1675-1681.

Southwood, T. R. E. 1988. Tactics, strategies and templets. Oikos 52: 3-18.

Steiner, N. C., and W. Köhler. 2003. Effects of landscape patterns on species richness: a modelling approach. Agriculture Ecosystems and Environment 98:353-361.

Stevens, V. M., A. Trochet, H. Van Dyck, J. Clobert, and M. Baguette. 2012. How is dispersal integrated in life histories: a quantitative analysis using butterflies. Ecology Letters 15:74-86.

Swihart, R. K., T. M. Gehring, M. B. Kolozsvary, and T. E. Nupp. 
2003. Responses of "resistant" vertebrates to habitat loss and fragmentation: the importance of niche breadth and range boundaries. Diversity and Distributions 9:1-18.

Swihart, R. K., J. J. Lusk, J. E. Duchamp, C. E. Rizkalla, and J. E. Moore. 2006. The roles of landscape context, niche breadth, and range boundaries in predicting species responses to habitat alteration. Diversity and Distributions 12:277-287.

Tilman, D. 1994. Competition and biodiversity in spatially structured habitats. Ecology 75:2-16.

Travis, J. M. J. 2003. Climate change and habitat destruction: a deadly anthropogenic cocktail. Proceedings of the Royal Society B: Biological Sciences 270:467-473.

Verberk, W., G. van der Velde, and H. Esselink. 2010. Explaining abundance-occupancy relationships in specialists and generalists: a case study on aquatic macroinvertebrates in standing waters. Journal of Animal Ecology 79:589-601.
Warren, M. S., J. K. Hill, J. A. Thomas, J. Asher, R. Fox, B. Huntley, D. B. Roy, et al. 2001. Rapid responses of British butterflies to opposing forces of climate and habitat change. Nature 414:65-69.

Weiner, J., and S. Xiao. 2012. Variation in the degree of specialization can maintain local diversity in model communities. Theoretical Ecology 5:161-166.

Wilson, D. S., and J. Yoshimura. 1994. On the coexistence of specialists and generalists. American Naturalist 144:692-707.

Yamauchi, A., and T. Miki. 2009. Intraspecific niche flexibility facilitates species coexistence in a competitive community with a fluctuating environment. Oikos 118:55-66.

Associate Editor: Mathew A. Leibold Editor: Judith L. Bronstein

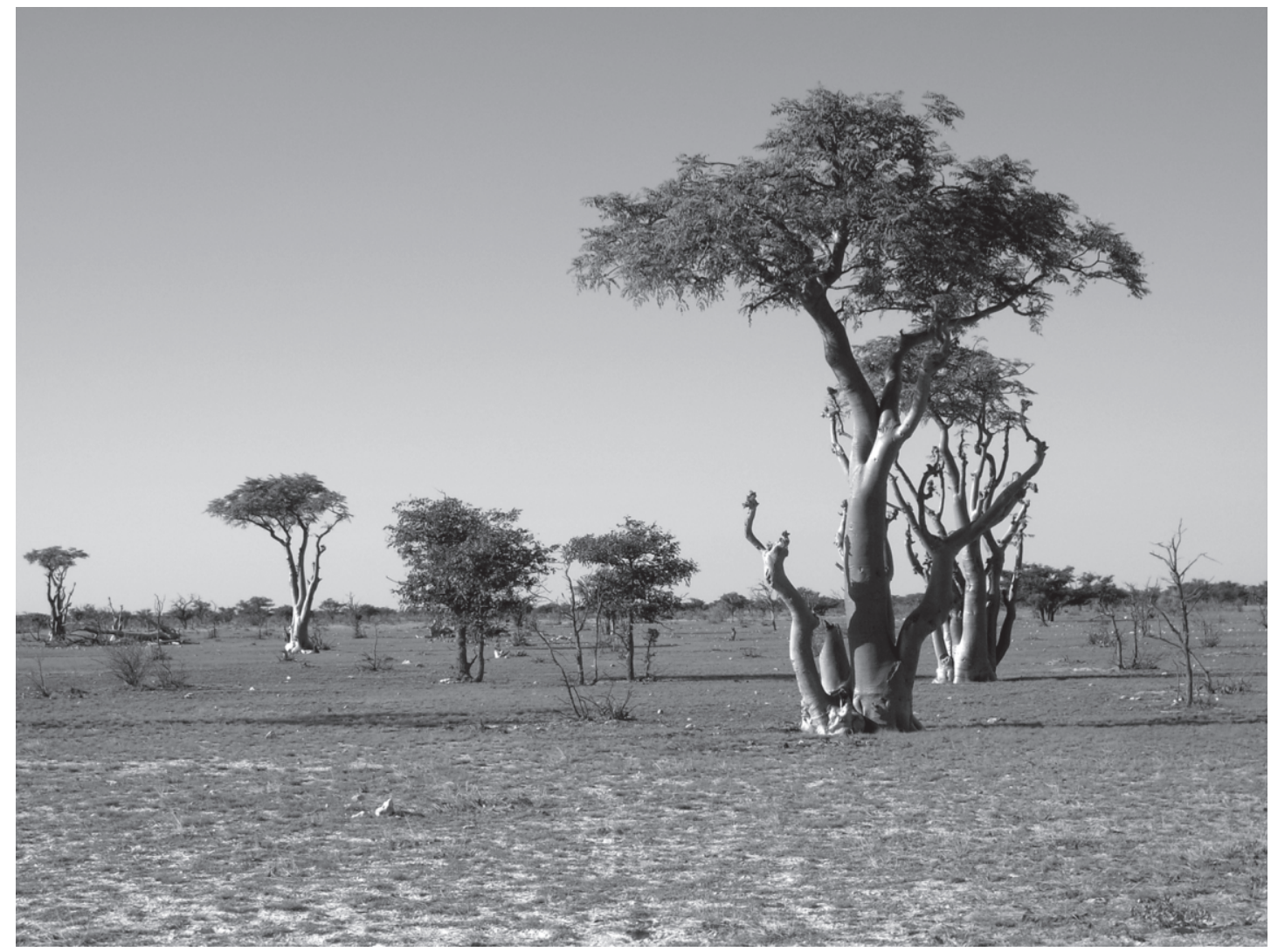

Ghost trees (Moringa ovalifolia) in Etosha National Park in Namibia. These trees are particularly specialized to the harsh environmental conditions experienced in this region. Photo credit: Séverine Vuilleumier. 